\title{
The dissipative structure of variational multiscale methods for incompressible flows
}

\author{
Javier Principe ${ }^{\mathrm{a}, *}$, Ramon Codina ${ }^{\mathrm{a}}$, Florian Henke ${ }^{\mathrm{b}}$ \\ ${ }^{a}$ Universitat Politècnica de Catalunya, Jordi Girona 1-3, Edifici C1, 08034 Barcelona, Spain \\ ${ }^{\mathrm{b}}$ Technische Universität München, Lehrstuhl für Numerische Mechanik, Boltzmannstrasse 15, D-85747 Garching b. München, Germany
}

\section{A R T I C L E I N F O}

\section{Article history:}

Received 16 January 2008

Received in revised form 25 July 2008

Accepted 4 September 2008

Available online 24 September 2008

\section{Keywords:}

Stabilized finite elements

Transient subscales

Orthogonal subscales

Energy transfer

\begin{abstract}
A B S T R A C T
In this paper, we present a precise definition of the numerical dissipation for the orthogonal projection version of the variational multiscale method for incompressible flows. We show that, only if the space of subscales is taken orthogonal to the finite element space, this definition is physically reasonable as the coarse and fine scales are properly separated. Then we compare the diffusion introduced by the numerical discretization of the problem with the diffusion introduced by a large eddy simulation model. Results for the flow around a surface-mounted obstacle problem show that numerical dissipation is of the same order as the subgrid dissipation introduced by the Smagorinsky model. Finally, when transient subscales are considered, the model is able to predict backscatter, something that is only possible when dynamic LES closures are used. Numerical evidence supporting this point is also presented.
\end{abstract}

(c) 2008 Elsevier B.V. All rights reserved.

\section{Introduction}

Variational multiscale (VMS) finite element methods for large eddy simulation (LES) of turbulence are promising. First attempts to apply the VMS idea to incompressible flow problems were made in $[17,18]$, where both small and large scales were solved and the classical LES filters were applied only to the small scales. The idea of simply using the algebraic approximation of the subscales, without any additional ingredient (e.g. without physical-based subgrid modeling), was introduced in [7] and elaborated in [5,19]. Very good results were obtained for fully developed and transitional turbulent flows. For a complete presentation in the context of isogeometric analysis, including results of homogeneous turbulence and turbulent channel flow, we refer the reader to [2]. It is important to note, however, that the algebraic approximation to the subscales in $[7,5,19]$ and in this work includes terms additional to those appearing in the classical GLS/SUPG methods [5,9].

Many comments have been and are usually made on the importance of the numerical scheme when a LES of a turbulent flow is performed. Chapter 7 of [26] is devoted to the numerical solution of the LES equations and several results are mentioned. The influence of the numerical scheme and its interaction with classical LES models was analyzed in [12], where truncation errors are compared with the amplitude of the subgrid terms and found to be dominant in many cases. The solution suggested in [12] is either to increase the accuracy of the scheme or to use the "pre-filtering" technique (to keep the filter size constant while the mesh size is

\footnotetext{
* Corresponding author.

E-mail addresses: principe@cimne.upc.edu (J. Principe), ramon.codina@upc.edu (R. Codina), henke@lnm.mw.tum.de (F. Henke).
}

reduced until $h$-convergence is achieved). As mentioned in [26], numerical experiments presented in [21] show that "the effect of subgrid models is completely or partially masked by the numerical error when second-order accurate methods are employed". The use of high order accurate schemes in order to minimize numerical dissipation is not an uncommon advice. It is also quite common to describe a numerical method according to how dissipative it is. However, precise measures of this property have not been presented up to date.

The first attempt to estimate numerical and subgrid dissipation was made in [11], where several schemes for LES of compressible flows are compared. The numerical dissipation is linked to the leading terms of the truncation error and an intuitive definition is presented. This definition is based on the difference between the discrete convective term and that given by a reference centered scheme of one order of accuracy higher. A scheme is considered suitable for LES if either the numerical dissipation is much lower than the subgrid one (condition $\mathrm{C} 1$ ) or the numerical dissipation is able to mimic the subgrid one (condition $\mathrm{C} 2$ ). The general sad conclusion is that neither condition $\mathrm{C} 1$ nor condition $\mathrm{C} 2$ are satisfied for the schemes analyzed.

Further analyses have been made in [10], where a method to compute the effective numerical dissipation is developed based on a finite difference approximation of the energy balance equation. The method is used to evaluate the dissipation in the context of the monotonically integrated LES approach, first proposed in [3], in which the Navier-Stokes equations are directly discretized without introducing neither the filtering operation nor the SGS stress tensor. The objective in [10] is to link the properties of the numerical method with the physics of turbulence by comparing numerical dissipation with the predictions of turbulence theory. 
A Galerkin least squares multiscale formulation of compressible flows was presented in [23]. In this reference the original multiscale formulation of LES introduced in [17] is advocated and the small scales are computed and filtered. The numerical dissipation introduced by the least squares terms, obtained multiplying the stabilization terms in the final algebraic system by the unknown, is greater than the subgrid dissipation for the closures analyzed. A similar approach is followed in [28] and the global conservation of energy is used to define the numerical dissipation introduced by the SUPG scheme (even the SUPG stress, an analog to the subgrid stress, is defined). A dynamic Smagorinsky model is used and the interaction with the dissipation introduced by the SUPG scheme is analyzed. Results of channel flow simulations show the numerical dissipation to be smaller than the physical one but still important. These results are used to propose a correction to the physical model to take into account the numerical scheme. The basic idea is that, ideally, the numerical scheme should not affect the physical model and therefore the proposal of $[281$ is to modify the physical model reducing the dissipation it introduces by the same amount the SUPG dissipation provides. This approach is opposed to that advocated in [3] and in the present work.

The global energy balance of the finite element component is also the starting point in [4] where the interior penalty method is analyzed and the quality of the solution is evaluated in terms of the numerical dissipation. In [13] it is shown that the numerical dissipation provided by the orthogonal subgrid scale method of Codina [7] provides a rate of transfer of subgrid kinetic energy proportional to the molectilar physica
appioprate choice of the stabilization ing in principle the need of introducir model.

In this article we introduce a loca dissipation for the variational multiscale
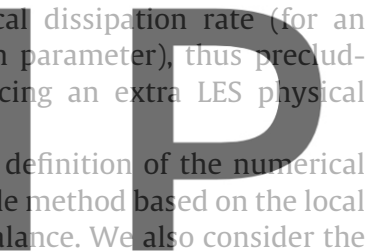
energy balance of the subgrid scale component, which permits us to clearly identify the energy transfer mechanisms. In this frame-

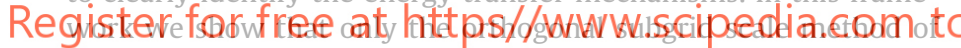
Codina [7] permits a proper separation of scales, in the sense that if a non-orthogonal projection is used, temporal derivatives couple the energy balance for the coarse and fine scale components. When the time dependent subscales of [9] are used, the model is capable of predicting backscatter (energy transfer from small to coarse scales).

The paper is organized as follows. In Section 2 the problem is stated, and in Section 3 its two scale approximation introduced. The core of the paper is presented in Section 4, where the energy budget in a region is discussed. Section 5 presents the numerical simulation of the flow over a surface mounted obstacle, where the different dissipation mechanisms can be observed. Some final conclusions close the paper in Section 6 .

\section{Problem statement}

Let us consider the flow of an incompressible fluid in a domain $\Omega \subset \mathbb{R}^{d}(d=2,3)$ with boundary $\Gamma=\partial \Omega$ during the time interval $[0, T]$. Let $\boldsymbol{u}: Q \rightarrow \mathbb{R}^{d}$ be the velocity field and $p: Q \rightarrow \mathbb{R}$ the pressure, with $Q=\Omega \times(0, T)$. The incompressible Navier-Stokes equations for $\boldsymbol{u}$ and $p$ can be written as

$\partial_{t} \boldsymbol{u}+\boldsymbol{u} \cdot \nabla u+\nabla p-v \nabla^{2} \boldsymbol{u}=\boldsymbol{f}$,

$\boldsymbol{\nabla} \cdot \boldsymbol{u}=0$,

where $\boldsymbol{f}$ is the vector of external forces and $v$ is the kinematic viscosity. Eqs. (1) and (2) must be supplemented with appropriate boundary and initial conditions. For simplicity in the presentation, only homogeneous Dirichlet boundary conditions will be considered.
We will also consider the LES problem that is found by applying a filter of the form

$\overline{\boldsymbol{v}}(\boldsymbol{x})=\int \boldsymbol{v}\left(\boldsymbol{x}^{\prime}\right) G\left(\boldsymbol{x}, \boldsymbol{x}^{\prime}\right) \mathrm{d} \boldsymbol{x}^{\prime}$,

to the Navier-Stokes Eqs. (1) and (2) for an appropriate filter function $G$. This operation results in an extra term: the divergence of the subgrid stress tensor. The LES problem consists in finding the filtered velocity field $\overline{\boldsymbol{u}}$ and the pressure field $\overline{\boldsymbol{p}}$ such that

$$
\begin{aligned}
& \partial_{t} \overline{\boldsymbol{u}}+\overline{\mathbf{u}} \cdot \nabla \overline{\boldsymbol{u}}+\nabla \overline{\boldsymbol{p}}-v \nabla^{2} \overline{\boldsymbol{u}}+\nabla \cdot \tau=\boldsymbol{f}, \\
& \nabla \cdot \overline{\boldsymbol{u}}=0,
\end{aligned}
$$

where $\tau$ is the residual stress tensor defined in components by $\tau_{i j}=\overline{\boldsymbol{u}_{i} \boldsymbol{u}_{j}}-\overline{\boldsymbol{u}}_{i} \overline{\boldsymbol{u}}_{j}$

Let $k=\frac{1}{2} \boldsymbol{u} \cdot \boldsymbol{u}$ be the pointwise kinetic energy. A kinetic energy conservation statement for the Navier-Stokes problem can be found by multiplying (1) by the velocity $u$. Using (2) one obtains
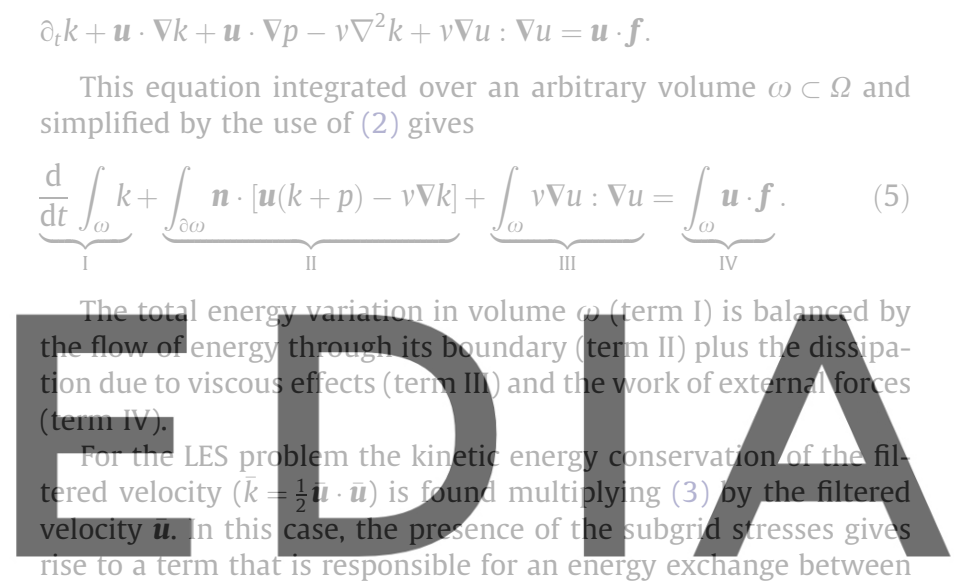
large scales (represented by filtered variables) and small scales

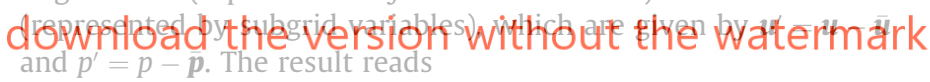

$\partial_{t} \bar{k}+\overline{\boldsymbol{u}} \cdot \nabla \bar{k}+\overline{\boldsymbol{u}} \cdot \nabla \bar{p}-v \nabla^{2} \bar{k}+v \nabla \overline{\boldsymbol{u}}: \nabla \overline{\boldsymbol{u}}+\nabla \cdot(\overline{\boldsymbol{u}} \cdot \tau)+\nabla \overline{\boldsymbol{u}}: \tau=\overline{\boldsymbol{u}} \cdot \bar{f} \cdot$

This equation integrated over an arbitrary volume $\omega$ and simplified by the use of (4) gives

$$
\begin{aligned}
& \underbrace{\frac{\mathrm{d}}{\mathrm{d} t} \int_{\omega}^{\bar{k}}}_{\mathrm{I}}+\underbrace{\int_{\partial \omega} \boldsymbol{n} \cdot[\overline{\boldsymbol{u}}(\bar{k}+\overline{\boldsymbol{p}})-v \nabla \bar{k}+\overline{\boldsymbol{u}} \cdot \tau]}_{\mathrm{II}}+\underbrace{\int_{\omega} v \nabla \overline{\boldsymbol{u}}: \nabla \overline{\boldsymbol{u}}}_{\mathrm{III}}+\underbrace{\int_{\omega} \nabla \overline{\boldsymbol{u}}: \tau}_{\mathrm{V}} \\
& =\underbrace{\int_{\omega} \overline{\boldsymbol{u}} \cdot \overline{\boldsymbol{f}}}_{\mathrm{IV}} .
\end{aligned}
$$

The interpretation of the terms corresponds to the meaning they had before, except for the new term $V$ that represents the transfer of energy between coarse and fine scales and for the last term in II which now includes the flow of energy through the boundary due to the work done by the mean velocity against the residual stress tensor. Note that this term comes from the filtering of the convective term and when the filter size tends to zero the residual stresses vanish and we recover (5). It is also possible to obtain a transport equation (and its integral form) for the subgrid kinetic energy $k^{\prime}=\frac{1}{2} \boldsymbol{u}^{\prime} \cdot \boldsymbol{u}^{\prime}$ in which term $V$ also appears [26].

In the discussion below we will consider the standard Smagorinsky LES model, in which the subgrid closure is

$\tau=v^{t} \nabla^{s} \overline{\boldsymbol{u}}$, 
where

$v^{t}=(c \Delta)^{2}\left(\nabla^{s} \overline{\boldsymbol{u}}: \nabla^{s} \overline{\boldsymbol{u}}\right)^{1 / 2}$.

In this expression, $c$ is a constant and $\Delta$ is the filter width. Let us remember that in this case term $V$ in (6) is positive (purely dissipative) and no backscatter of energy (transfer of energy from fine to coarse scales) can be predicted by the model. If this backscatter has to be simulated, constant $c$ has to be replaced by a quantity computed in terms of the flow variables, leading to the so called $d y$ namic versions of Smagorinsky model (see [26]).

\section{Variational multiscale approximation}

From the point of view of the discrete approximation, the incompressible Navier-Stokes problem (1), (2) and the LES problem (3) and (4) are identical except for the term $\nabla \cdot \tau$ in Eq. (3), and therefore the same discrete formulation is usually used. In this section we consider the incompressible Navier-Stokes problem that can be written in a weak form as follows: find $(\boldsymbol{u}, \boldsymbol{p}) \in \mathbb{V} \times \mathbf{Q}$ such that
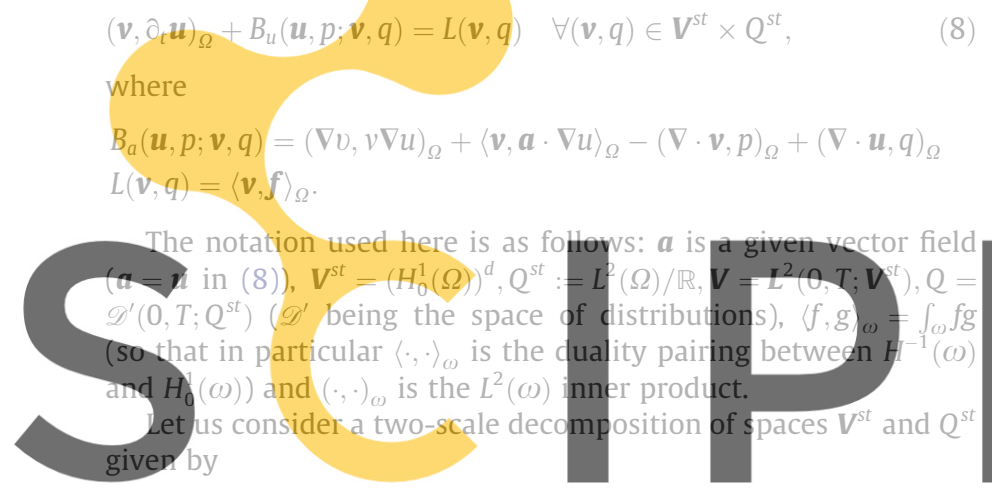

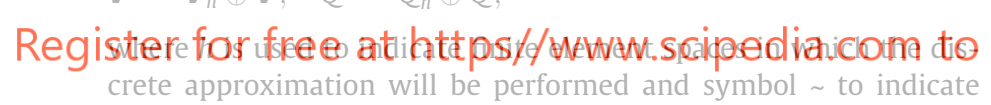
subgrid spaces. The latter are any completion of the former in the continuous spaces. Applied to the weak form of the probiem, this decomposition leads to

$$
\begin{aligned}
& \left(\boldsymbol{v}_{h}, \partial_{t} \boldsymbol{u}_{h}\right)_{\Omega}+B_{u}\left(\boldsymbol{u}_{h}, p_{h} ; \boldsymbol{v}_{h}, q_{h}\right)+\left(\boldsymbol{v}_{h}, \partial_{t} \tilde{\boldsymbol{u}}\right)_{\Omega}+B_{u}\left(\tilde{\boldsymbol{u}}, \tilde{p} ; \boldsymbol{v}_{h}, q_{h}\right)=L\left(\boldsymbol{v}_{h}, q_{h}\right) \\
& \left(\tilde{\boldsymbol{v}}, \partial_{t} \boldsymbol{u}_{h}\right)_{\Omega}+B_{u}\left(\boldsymbol{u}_{h}, p_{h} ; \tilde{\boldsymbol{v}}, \tilde{q}\right)+\left(\tilde{\boldsymbol{v}}, \partial_{t} \tilde{\boldsymbol{u}}\right)_{\Omega}+B_{u}(\tilde{\boldsymbol{u}}, \tilde{p} ; \tilde{\boldsymbol{v}}, \tilde{q})=L(\tilde{\boldsymbol{v}}, \tilde{q})
\end{aligned}
$$

which must hold for all $\left(\boldsymbol{v}_{h}, q_{h}\right) \in \boldsymbol{V}_{h} \times Q_{h}$ and for all $(\tilde{\boldsymbol{v}}, \tilde{q}) \in \tilde{\boldsymbol{V}} \times \widetilde{Q}$. The first equation governs the evolution of resolvable scales (the functions of the spaces $\boldsymbol{V}_{h}$ and $Q_{h}$ ) and has two terms on the left hand side: the first one is the Galerkin contribution and the second one takes into account the influence of the subgrid scale on the finite element components. After integration by parts within each element and neglecting interelement boundary terms we arrive at

$$
\begin{aligned}
& \left(\boldsymbol{v}_{h}, \partial_{t} \boldsymbol{u}_{h}\right)_{\Omega}+\left(\nabla v_{h}, v \nabla u_{h}\right)_{\Omega}+\left\langle\boldsymbol{v}_{h}, \boldsymbol{a} \cdot \nabla u_{h}\right\rangle_{\Omega} \\
& \quad-\left(\nabla \cdot \boldsymbol{v}_{h}, p_{h}\right)_{\Omega}+\left(q_{h}, \boldsymbol{\nabla} \cdot \boldsymbol{u}_{h}\right)_{\Omega}+\left(\boldsymbol{v}_{h}, \partial_{t} \tilde{\boldsymbol{u}}\right)_{\Omega} \\
& \quad+\sum_{K}\left(\mathscr{L}^{*} \boldsymbol{v}_{h}-\nabla q_{h}, \tilde{\boldsymbol{u}}\right)_{K}-\sum_{K}\left(\nabla \cdot \boldsymbol{v}_{h}, \tilde{p}\right)_{K}=\left\langle\boldsymbol{v}_{h}, \boldsymbol{f}\right\rangle_{\Omega},
\end{aligned}
$$

where $\mathscr{L}^{*}$ is the adjoint of the convection diffusion operator $\mathscr{L}$, defined as

$$
\mathscr{L}=-v \nabla^{2}+\boldsymbol{a} \cdot \nabla, \quad \mathscr{L}^{*}=-v \nabla^{2}-\boldsymbol{a} \cdot \nabla
$$

and $\boldsymbol{a}$ is the advection velocity that depends on the formulation considered (it has been considered solenoidal to obtain the above expressions).
Remark 1. The standard variational multiscale formulation for stabilization purposes, originally proposed in [20] and analyzed in [6], is motivated for the problem obtained with $\boldsymbol{a}=\boldsymbol{u}_{h}$. However, if the nonlinear tracking of subscales proposed in [9] is considered, we have $\boldsymbol{a}=\boldsymbol{u}_{h}+\tilde{\boldsymbol{u}}$. In this case we are considering all the terms that result from the splitting of the convective term. As shown in [9], analogous terms to the various stress types in the LES approach are recovered in a "natural" way from our pure numerical approach.

In [9] the subscale is considered a variable in its own right (similar to internal variables in solid mechanics) and it is assumed to be time dependent. It is found as the solution of the fine scale equation, which is approximated replacing the Navier-Stokes operator by two parameters $\tau_{m}^{-1}$ and $\tau_{c}^{-1}$ motivated below. This leads to the following fine scale problem posed on each element $K$ [9] (decoupling the problems is also part of the approximation):

$$
\begin{aligned}
& \partial_{t} \tilde{\boldsymbol{u}}+\tau_{m}^{-1} \tilde{\boldsymbol{u}}=\Pi\left(\boldsymbol{f}-\partial_{t} \boldsymbol{u}_{h}-\mathscr{L} \boldsymbol{u}_{h}-\nabla \boldsymbol{p}_{h}\right) \\
& \tau_{c}^{-1} \tilde{p}=\Pi\left(-\nabla \cdot \boldsymbol{u}_{h}\right)
\end{aligned}
$$

where $\Pi$ is the projection onto the space of subscales. The properties of the method depend strongly on the choice of the projection $\Pi$, that is, on the choice of the space of subscales. Two options are commonly considered. The first one takes $\Pi=I$, what is called algebraic subgrid scale (ASGS) in [6] (in fact, $\Pi$ needs to be the identity only when acting on functions of the form of the right-hand-side in (10), (11)). The second one is the orthogonal subscales (OSS) meth-

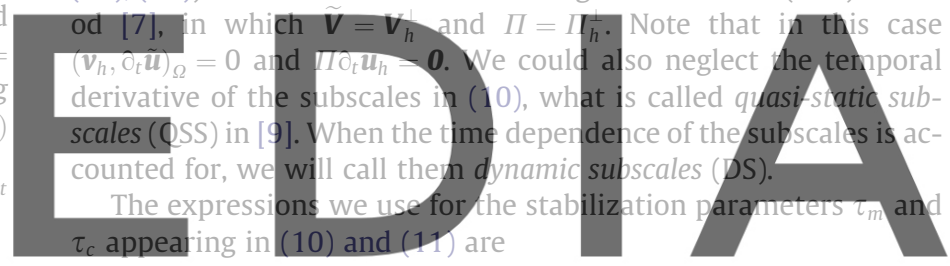

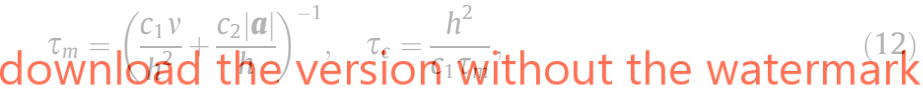
where $h$ is the mesh size and $c_{1}$ and $c_{2}$ are algorithmic constants whose values are taken as 4 and 2, respectively, for linear elements. This choice is based on numerical experience and one-dimensional analysis. Note that when the nonlinear tracking of the subscales of Codina et al. [9] is used (case in which $\boldsymbol{a}=\boldsymbol{u}_{h}+\tilde{\boldsymbol{u}}$ ), (10), (11) is nonlinear in $\tilde{\boldsymbol{u}}$ through $\tau_{m}$ and $\tau_{c}$. We devote the rest of the section to summarize the approach we follow to justify these expressions.

Let $\boldsymbol{U}=(\boldsymbol{u}, p)$ and consider the stationary Navier-Stokes operator

$$
\mathscr{N}(\boldsymbol{U}):=\left[\begin{array}{ll}
\mathscr{L}(\boldsymbol{u}) & \nabla p \\
\nabla \cdot \boldsymbol{u} & 0
\end{array}\right]
$$

where $\mathscr{L}(\boldsymbol{u})$ is the convection-diffusion operator introduced earlier. Our proposal is to approximate the operator $\mathscr{N}$ by an algebraic operator $\tau^{-1}$ so that $\|\mathscr{N}\|_{M} \leqslant\left\|\tau^{-1}\right\|_{M}$, where $\|\cdot\|_{M}$ is the operator norm induced by the $L^{2}$-norm scaled by a matrix $\boldsymbol{M}$. This matrix must be such that $\mathscr{N}(\boldsymbol{U})^{\mathrm{t}} \boldsymbol{M} \mathscr{N}(\boldsymbol{U})$ is dimensionally well defined. Different choices are possible, and a particularly suited one is introduced later. The condition $\|\mathscr{N}\|_{M} \leqslant\left\|\tau^{-1}\right\|_{M}$ means that the energy of the exact subscales does not exceed the energy of the approximated subscales, as shown in [8] in the context of shallow waters. The closer $\|\mathscr{N}\|_{M}$ is to $\left\|\tau^{-1}\right\|_{M}$, the closer these energies are. In the scalar convection diffusion problem [7], it is possible to show that the norm of the approximated and the exact subscales are approximately equal. In can be shown using an approximate Fourier analysis [8] that condition $\|\mathscr{N}\|_{M} \leqslant\left\|\tau^{-1}\right\|_{M}$ can be satisfied if $\tau^{-1}$ is chosen such that

$\left\|\tau^{-1}\right\|_{M}=\left\|\widehat{\mathscr{N}}\left(\boldsymbol{k}_{0}\right)\right\|_{M}$ 
where $\widehat{\mathscr{N}}\left(\boldsymbol{k}_{0}\right)$ is the Fourier transform of the operator $\mathscr{N}$ computed with a fixed velocity and evaluated at an unknown wavenumber $\boldsymbol{k}_{0}$ whose existence is guaranteed by the mean value theorem.

In practice we impose condition (13) by computing the spectrum with respect to $\boldsymbol{M}^{-1}$ of $\tau^{-1} \boldsymbol{M} \tau^{-1}$ and of $\widehat{\mathscr{N}}^{*}\left(\boldsymbol{k}_{0}\right) \boldsymbol{M} \widehat{\mathscr{N}}\left(\boldsymbol{k}_{0}\right)$ and imposing the equality of the largest eigenvalues. Actually, the ideal situation is found when both matrices have the same spectrum, so that $\tau^{-1}$ is a better approximation to $\widehat{N}\left(\boldsymbol{k}_{0}\right)$. We omit the subscript in $\boldsymbol{k}_{0}$ in what follows.

In our case, when $d=2$ we have

$$
\widehat{N}(\boldsymbol{k})=\left[\begin{array}{ccc}
v \kappa^{2}+\mathrm{i} \kappa_{j} a_{j} & 0 & \mathrm{i} \kappa_{1} \\
0 & v \kappa^{2}+\mathrm{i} \kappa_{j} a_{j} & \mathrm{i} \kappa_{2} \\
\mathrm{i} \kappa_{1} & \mathrm{i} \kappa_{2} & 0
\end{array}\right],
$$

where $\kappa_{j}=k_{j} / h, \kappa=|\boldsymbol{k}| / h$ and $\mathrm{i}=\sqrt{-1}$. Taking a scaling matrix $\boldsymbol{M}=\operatorname{diag}\left(\mu_{u}, \mu_{u}, \mu_{p}\right)$ the eigenvalues of $\widehat{\mathscr{N}}^{*}(\boldsymbol{k}) \boldsymbol{M} \widehat{\mathscr{N}}(\boldsymbol{k})$ with respect to $\boldsymbol{M}^{-1}$ are

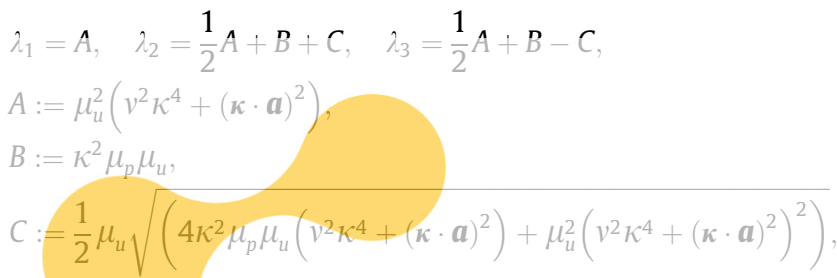

and assuming $\tau=\operatorname{diag}\left(\tau_{m}, \tau_{m}, \tau_{c}\right)$ those of $\tau^{-1} \boldsymbol{M} \tau^{-1}$ are $\mu_{u}^{2} / \tau_{m}^{2}, \mu_{u}^{2} / \tau_{m}^{2}$, and $\mu_{n}^{2} / \tau_{c}^{2}$. It can be easily shown that taking the scaling $\mu_{\mathrm{u}}=\left(v^{2} \kappa^{4}+(\boldsymbol{k} \cdot \boldsymbol{a})^{2}\right)^{-1}$ and $\mu_{p}=2 \kappa^{-2}$ the spectrum of both matrices is identical, a condition stronger than (13) which implies that
the energy of the exact and the approximated subscales are approx-
intately the same. In this case
$\tau_{m}=\left(v^{2} \kappa^{4}+(\boldsymbol{k} \cdot \boldsymbol{a})^{2}\right)^{-1 / 2}, \quad \tau_{c}=\frac{\boldsymbol{\kappa}^{-2}}{\tau_{m}}$,
which behave in terms of $h, \boldsymbol{a}$ and $v$ as the expressions given in (12). In principle, the expression of the parameters in (12) changes from point to point. In order to simplify the notation, we shall con-

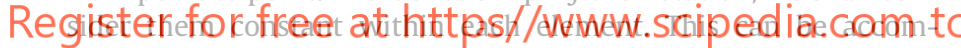
plished, for example, taking as advection velocity the mean value of $\boldsymbol{a}$ within each element.

\section{Local energy conservation}

Following [15] we can write local conservation statements in domains $\omega \subset \Omega$ formed by arbitrary patches of elements introducing a discrete approximation of the fluxes $\boldsymbol{h}^{\omega}: \partial \omega \rightarrow \mathbb{R}^{\mathrm{d}}$, denoted as $\boldsymbol{h}_{h}^{\omega}$, that satisfies

$$
\begin{aligned}
& \left(\boldsymbol{v}_{h}, \partial_{t} \boldsymbol{u}_{h}\right)_{\omega}+\left(\nabla v_{h}, v \nabla u_{h}\right)_{\omega}+\left\langle\boldsymbol{v}_{h}, \boldsymbol{a} \cdot \nabla u_{h}\right\rangle_{\omega}-\left(\nabla \cdot \boldsymbol{v}_{h}, p_{h}\right)_{\omega} \\
& \quad+\left(q_{h}, \nabla \cdot \boldsymbol{u}_{h}\right)_{\omega}+\left(\Pi \boldsymbol{v}_{h}, \partial_{t} \tilde{\boldsymbol{u}}\right)_{\omega}+\sum_{K \subset \omega}\left(\Pi\left(\mathscr{L}^{*} \boldsymbol{v}_{h}-\nabla q_{h}\right), \tilde{\boldsymbol{u}}\right)_{K} \\
& \quad-\sum_{K \subset \omega}\left(\Pi \nabla \cdot \boldsymbol{v}_{h}, \tilde{p}\right)_{K}=\left\langle\boldsymbol{v}_{h}, \boldsymbol{f}\right\rangle_{\omega}+\left\langle\boldsymbol{v}_{h}, \boldsymbol{h}_{h}^{\omega}\right\rangle_{\partial \omega} .
\end{aligned}
$$

Note that we have introduced the projection $\Pi$ in the terms coming from the subgrid scales. The formulation of the discrete problem (9) is unaffected by this addition because $(\boldsymbol{v}, \tilde{\boldsymbol{u}})_{\Omega}=$ $(\Pi \boldsymbol{v}, \tilde{\boldsymbol{u}})_{\Omega} \forall \boldsymbol{v} \in \boldsymbol{V}$. However this is not the case in a subdomain $\omega$ of the domain $\Omega$ because the introduction of the projection will change the recovered fluxes $\boldsymbol{h}_{h}^{(\omega)}$.

Taking $\boldsymbol{v}_{h}=\boldsymbol{u}_{h}$ and $q_{h}=p_{h}$ in (14) we arrive at a local energy conservation statement

$$
\begin{aligned}
& \frac{1}{2} d_{t}\left\|\boldsymbol{u}_{h}\right\|_{\omega}^{2}+v\left\|\nabla u_{h}\right\|_{\omega}^{2}+\left\langle\boldsymbol{u}_{h}, \boldsymbol{a} \cdot \nabla u_{h}\right\rangle_{\omega}+\left(\Pi \boldsymbol{u}_{h}, \partial_{t} \tilde{\boldsymbol{u}}\right)_{\omega} \\
& \quad+\sum_{K \subset \omega}\left(\Pi\left(\mathscr{L}^{*} \boldsymbol{u}_{h}-\nabla p_{h}\right), \tilde{\boldsymbol{u}}\right)_{K}-\sum_{K \subset \omega}\left(\Pi \nabla \cdot \boldsymbol{u}_{h}, \tilde{p}\right)_{K} \\
& \quad=\left\langle\boldsymbol{u}_{h}, \boldsymbol{f}\right\rangle_{\omega}+\left\langle\boldsymbol{u}_{h}, \boldsymbol{h}_{h}^{\omega}\right\rangle_{\partial \omega},
\end{aligned}
$$

where $d_{t}=\mathrm{d} / \mathrm{d} t$. As done with Eqs. (5) and (6), a physical interpretation of the different terms in equation (15) can be made. The first term on the left-hand-side represents the temporal variation of the total kinetic energy of the finite element scales in the volume $\omega$, which occurs because of the viscous dissipation represented by the second term on the left-hand-side and the work done against external forces represented by the first term of the right-hand-side. They represent the same effects as terms I, III and IV in (6). The second term on the right-hand-side represents (part of) the work done on the control surface $\partial \omega$. The fluxes $\boldsymbol{h}_{h}^{\omega}$ are the variationally consistent tractions [15]: if the continuous problem is considered we have

$\boldsymbol{h}^{\omega}=-p \boldsymbol{n}+v \boldsymbol{n} \cdot \nabla u$,

and then

$\left\langle\boldsymbol{u}, \boldsymbol{h}^{\omega}\right\rangle_{\partial \omega}=\int_{\partial \omega} \boldsymbol{n} \cdot[\boldsymbol{u} p-v \nabla k]$,

which corresponds to part of term II in (6). Term II in (6) also contains the flux due to convective effects. In the non-conservative form of the convective term considered so far we have

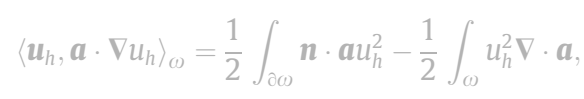

whereas in conservative form we have

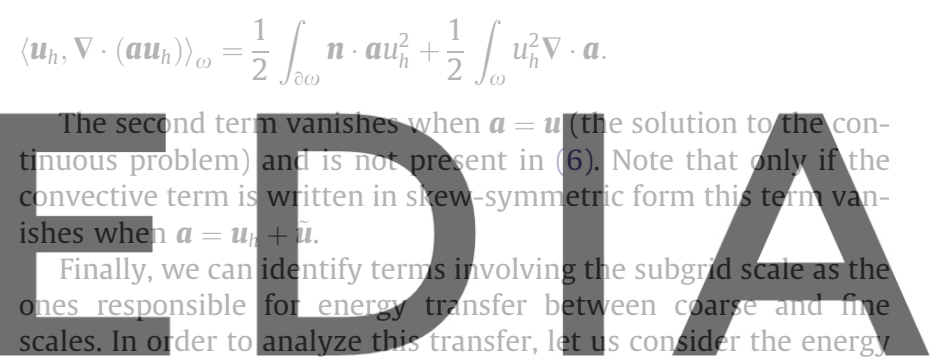

balance of the subgrid component. Multiplying (10) by $\tilde{u}$ and (11) by $\tilde{p}$ and integrating in the domain $\omega$ we have

\section{download the version without the watermark} $\frac{1}{2} d_{t}\|\tilde{\boldsymbol{u}}\|_{\omega}^{2}+\tau_{m}^{-1}\|\tilde{\boldsymbol{u}}\|_{\omega}^{2}+\tau_{c}^{-1}\|\tilde{p}\|_{\omega}^{2}+\left(\Pi \partial_{t} \boldsymbol{u}_{h}, \tilde{\boldsymbol{u}}\right)_{\omega}$

$$
+\sum_{K \subset \omega}\left(\Pi\left(\mathscr{L} \boldsymbol{u}_{h}+\nabla p_{h}\right), \tilde{\boldsymbol{u}}\right)_{K}+\sum_{K \subset \omega}\left(\Pi \nabla \cdot \boldsymbol{u}_{h}, \tilde{p}\right)_{K}=\sum_{K \subset \omega}(\Pi f, \tilde{\boldsymbol{u}})_{K} .
$$

In this case the first term on the left-hand-side represents the temporal variation of the subgrid kinetic energy in the volume $\omega$, which occurs because of the dissipation represented by the second and third terms on the left-hand-side and the work done against external forces represented by the term of the right-hand-side. Again, as in (15) we can identify the terms involving the finite element components of the velocity and pressure as the ones responsible for energy transfers between fine and coarse scales.

The first important conclusion we can draw from the inspection of (15) and (16) is that only if the orthogonal subscales are considered a proper separation of scales is achieved, because only in this case the finite element energy budget is independent of the subscale temporal derivative, and likewise the subscale energy budget is independent of the finite element temporal derivative. Adding up (15) and (16) we observe that the variation of the total kinetic energy is equal to the variation of the kinetic energy of the finite element scales plus the variation of the kinetic energy of the subgrid scales, and likewise the dissipation is equal to the dissipation of the finite element scales plus the dissipation of the subgrid scales.

Therefore, if we consider orthogonal subscales we can define the numerical dissipation of the finite element kinetic energy as

$\varepsilon_{h}=\sum_{K \subset \omega}\left(\Pi\left(\mathscr{L}^{*} \boldsymbol{u}_{h}-\nabla p_{h}\right), \tilde{\boldsymbol{u}}\right)_{K}-\sum_{K \subset \omega}\left(\Pi \nabla \cdot \boldsymbol{u}_{h}, \tilde{p}\right)_{K}$, 
and the production of subgrid energy as

$P_{s}=\sum_{K \subset \omega}\left(\Pi\left(\mathscr{L} \boldsymbol{u}_{h}+\nabla p_{h}\right), \tilde{\boldsymbol{u}}\right)_{K}+\sum_{K \subset \omega}\left(\Pi \nabla \cdot \boldsymbol{u}_{h}, \tilde{p}\right)_{K}$.

Let us postpone for a moment the justifications of the names of dissipation and production and let us concentrate on their relation first. Definitions (17) and (18) immediately imply

$\varepsilon_{h}+P_{s}=\sum_{K \subset \omega}\left(\Pi\left(\mathscr{L}^{*} \boldsymbol{u}_{h}+\mathscr{L} \boldsymbol{u}_{h}\right), \tilde{\boldsymbol{u}}\right)_{K}$

Therefore, independently of the space of subscales considered (and therefore independently of the choice of projection $\Pi$ ), adding up Eqs. (15) and (16) we have

$$
\begin{aligned}
\frac{1}{2} d_{t} & \left\|\boldsymbol{u}_{h}+\tilde{\boldsymbol{u}}\right\|_{\omega}^{2}+\left\langle\boldsymbol{u}_{h}, \boldsymbol{a} \cdot \nabla u_{h}\right\rangle_{\omega}+v\left\|\nabla u_{h}\right\|_{\omega}^{2}+\tau_{m}^{-1}\|\tilde{\boldsymbol{u}}\|_{\omega}^{2}+\tau_{c}^{-1}\|\tilde{p}\|_{\omega}^{2} \\
& +\sum_{K \subset \omega}\left(\Pi\left(\mathscr{L}^{*} \boldsymbol{u}_{h}+\mathscr{L} \boldsymbol{u}_{h}\right), \tilde{\boldsymbol{u}}\right)_{K} \\
= & \sum_{K \subset \omega}(\Pi \boldsymbol{f}, \tilde{u})_{K}+\left\langle\boldsymbol{u}_{h}, \tilde{\boldsymbol{f}}\right\rangle_{\omega}+\left\langle\boldsymbol{u}_{h}, \tilde{h}_{h}^{(\omega)}\right\rangle_{\partial \omega},
\end{aligned}
$$

that is an equation for the total kinetic energy. Energy transfer between finite element components and subscales vanish when (15) and (16) are added up, since one transfer term is equal to minus the other, as it should, except for the viscous term, which could be either neglected in the orthogonal projection (see the comments in [7]) or easily controlled by the viscous dissipation of the finite element component.

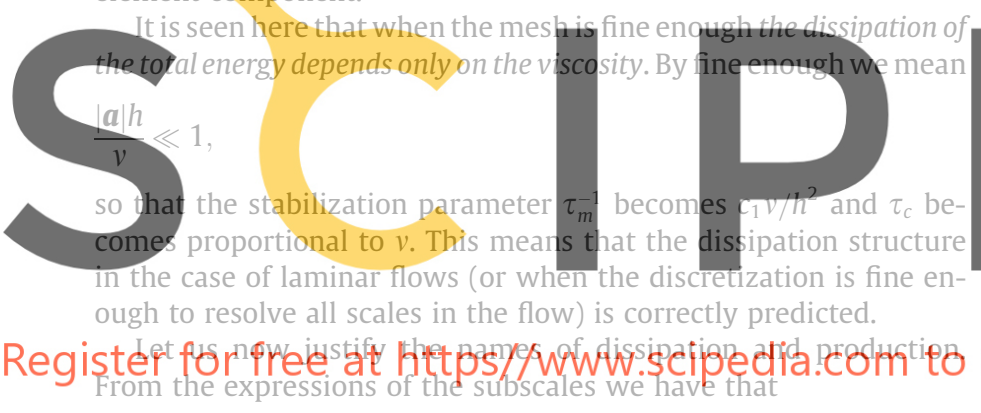

$\tilde{\boldsymbol{u}}=\tau_{m} \Pi\left(-\mathscr{L} \boldsymbol{u}_{h}-\nabla p_{h}\right)+\tau_{m} \Pi \boldsymbol{f}-\tau_{m} \partial_{t} \tilde{\boldsymbol{u}}$

$\tilde{p}=-\tau_{c} \Pi\left(\nabla \cdot \boldsymbol{u}_{h}\right)$,

and therefore

$$
\begin{aligned}
\varepsilon_{h}= & \underbrace{\sum_{K \subset \omega} \tau_{m}\left(\Pi\left(-\mathscr{L}^{*} \boldsymbol{u}_{h}+\nabla p_{h}\right), \Pi\left(\mathscr{L} \boldsymbol{u}_{h}+\nabla p_{h}\right)\right)_{K}+\sum_{K \subset \omega} \tau_{c}\left\|\Pi \nabla \cdot \boldsymbol{u}_{h}\right\|_{K}^{2}}_{\text {II }} \\
& +\underbrace{\sum_{K \subset \omega} \tau_{m}\left(\Pi\left(\mathscr{L}^{*} \boldsymbol{u}_{h}-\nabla p_{h}\right), \Pi \boldsymbol{f}\right)_{K}}_{\text {III }} \\
& +\underbrace{\sum_{K \subset} \tau_{m}\left(\Pi\left(-\mathscr{L}^{*} \boldsymbol{u}_{h}+\nabla p_{h}\right), \partial_{t} \tilde{\boldsymbol{u}}\right)_{K}}_{K \subset \omega}
\end{aligned}
$$

Let us discuss the implications of the three terms underbraced:

- Term I provides numerical stability and, from a more physical standpoint, dissipation strictly speaking. For linear elements it is given by

$$
\sum_{K \subset \omega} \tau_{m}\left\|\Pi\left(\boldsymbol{a} \cdot \nabla u_{h}+\nabla p_{h}\right)\right\|_{K}^{2}+\sum_{K \subset \omega} \tau_{c}\left\|\Pi \nabla \cdot \boldsymbol{u}_{h}\right\|_{K}^{2}
$$

When higher order elements are used the first term in I could contain a negative contribution due to the viscous differential operator, but this contribution is known to be smaller than the viscous dissipation $v\left\|\nabla u_{h}\right\|_{K}^{2}$ on each element provided the constant $c_{1}$ in the definition of the stabilization parameters (12) is big enough [7]. That the first term in I provides physical dissipation is more than a conjecture. Let us write
$\tau_{m}\left\|\Pi\left(\boldsymbol{a} \cdot \nabla u_{h}+\nabla p_{h}\right)\right\|_{K}^{2}=\int_{K} \varepsilon_{\text {num }}$

Let us consider a statistical treatment of the flow and let $\langle\cdot\rangle$ be now the ensemble average (or time average, for fully developed turbulent flows). If $1 / h$ is assumed to lie in the inertial range of the Kolmogorov energy cascade and the classical assumptions of statistical fluid mechanics apply, it is shown in [13] that when $v \rightarrow 0$

$\left\langle\varepsilon_{\text {num }}\right\rangle \propto\left\langle v|\nabla \boldsymbol{u}|^{2}\right\rangle$,

where $\boldsymbol{u}$ is the solution of the continuous problem (reference [13] deals with the global energy balance but the same arguments can be applied to the local definition presented here). Therefore, the numerical dissipation $\varepsilon_{\text {num }}$ is statistically proportional to the molecular dissipation of the flow. This is precisely the requirement posed by Lilly for LES models [24] to show that

$\left\langle\varepsilon_{\text {les }}\right\rangle \propto\left\langle v|\nabla \boldsymbol{u}|^{2}\right\rangle$,

where

$\varepsilon_{\text {les }}=v^{t}|\nabla \overline{\boldsymbol{u}}|^{2}$

The introduction of the local definition of numerical dissipation permits to develop a strategy for comparing LES and numerical based approaches to turbulence simulation. If we have a sufficiently accurate solution of a given flow (obtained by performing a direct numerical simulation) we could construct a discretization of size $h$ such that $h^{-1}$ lies in the inertial range and then compute

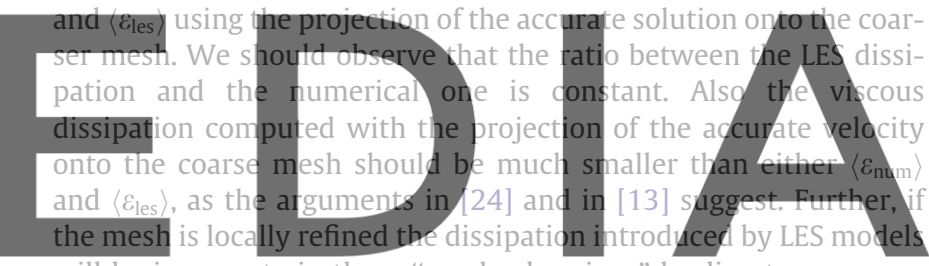
will be inaccurate in these "resolved regions" leading to an excessive dissipation and a deterioration of the convergence rate [16].

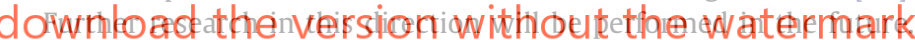
The previous argument suggests that LES modeling is completely redundant in our pure numerical approach because the numerical dissipation already presents the correct structure if the discretization is such that $h^{-1}$ lies in the inertial range. Moreover, it also presents the correct structure when the mesh is refined. In particular, laminar flows are correctly predicted by this approach provided the LES modeling is not considered, as shown before. However, we would like to stress that these conclusions are only valid when the hypothesis made in [24] and in [13] are satisfied, and this excludes many flow regimes where strong anisotropies are important, such as shear or boundary layers (and in this case it is known that a dumping of the Smagorinsky eddy viscosity is necessary). Therefore, the question of whether it is necessary or not to use a LES model together with the formulation we have presented is hard and requires the analysis of the predictions of both models in several different flows. Nevertheless, as shown in the numerical experiment of Section 5, our formulation is able to capture turbulent features of the flow, at least regarding mean values (see also $[2,14,27]$ and the discussion in [26]).

- Term II could be negative and provide transfer of energy to the finite element scales if the projection of the external force on the space of subscales does not vanish. It is the mechanism by which the flow could be excited by a highly spatially oscillating force.

- Term III, only present when the time dependent subscales introduced in [9] are used, could provide a backscatter of energy, that is to say, transfer of energy from the subgrid scales to the finite element scales. Note that the dependence of the dissipation on the temporal derivatives is only apparent. It comes from (19), 

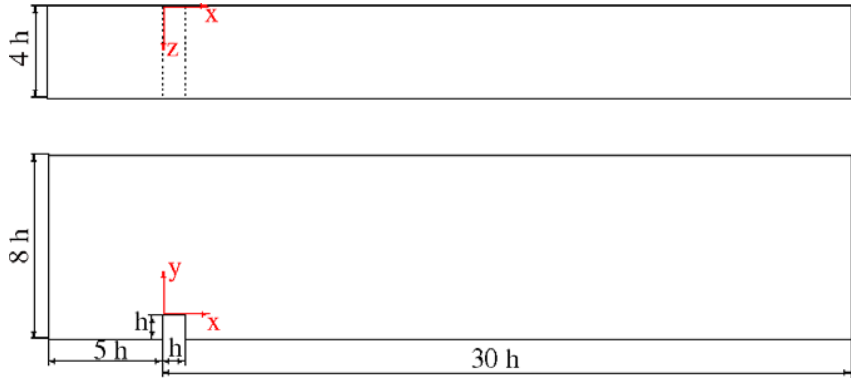

Fig. 1. Geometry of the surface-mounted obstacle problem (top: top view; bottom: front view).

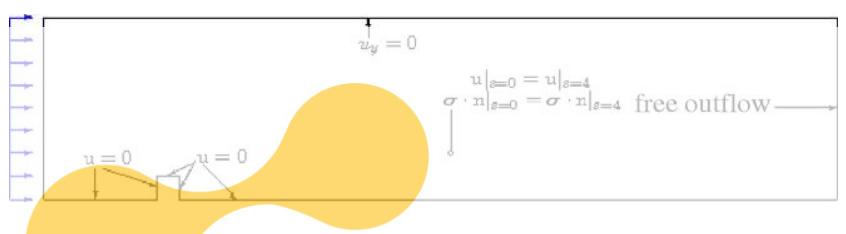

Fig. 2. Boundary conditions of the surface-mounted obstacle problem.
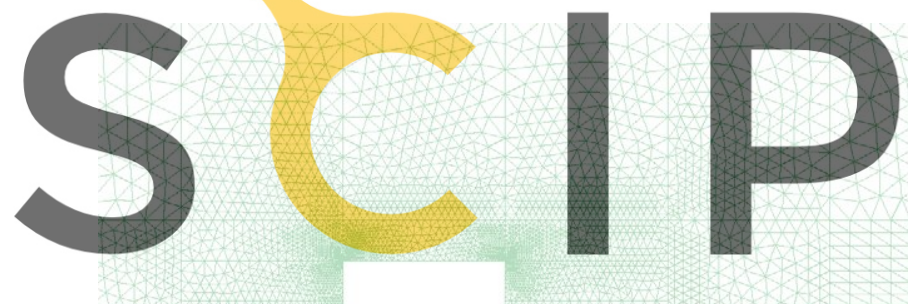

Register for free at https//www.scipedia.com

Fig. 3. Closeup view of the mesh around the obstacle. where $\tilde{\boldsymbol{u}}$ is expressed in terms of the residual of the finite element component and of $\partial_{t} \tilde{\boldsymbol{u}}$. So, at the end, expression (17) depends on the subscales at each time instant. It is important to note that more advanced physical-based subgrid models such as the dynamic Smagorinsky model are also able to predict backscatter. Again, as mentioned in the analysis of term I, the only definitive test would be a direct numerical simulation (DNS) to decide if the spatial structure of this contribution correctly represents the physical phenomena.

As a summary of the developments presented in this section, we have introduced a local definition of numerical dissipation and we have identified the mechanism of energy transfer between coarse and fine scales. The first point permits to develop a strategy to evaluate the potential use of purely numerical approaches to the simulation of turbulence and gives rise to the second point, the description of the energy transfer. The subgrid scale problem is driven by the (projection of the) residual of the mean flow, and the work done by the subscales against this "external" force is the source of energy (of the fine scale problem). This energy given to the fine scale is spent by the mean flow to drive the subgrid scale. The part of this energy transfer that depends on the pressure (and the viscosity if higher order elements are used) could be considered as "numerical" and the part that depends on the nonlinear term as "physical", as explained by the Kolmogorov theory. Nevertheless, we are strongly opposed to this artificial separation.
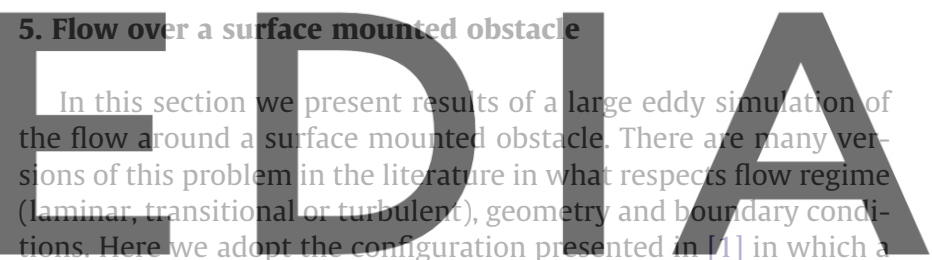

cuboid-shaped obstacle with a square cross-profile (of side $h$ ) is placed gn a surface, spanning the entice width as shown in Fig. 1

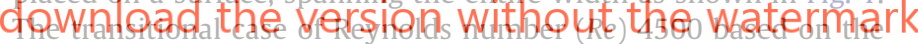
inflow velocity and obstacle height is considered.

Boundary conditions are illustrated in (Fig. 2) (there and in what follows lengths are always specified in terms of $h$ ). The fluid
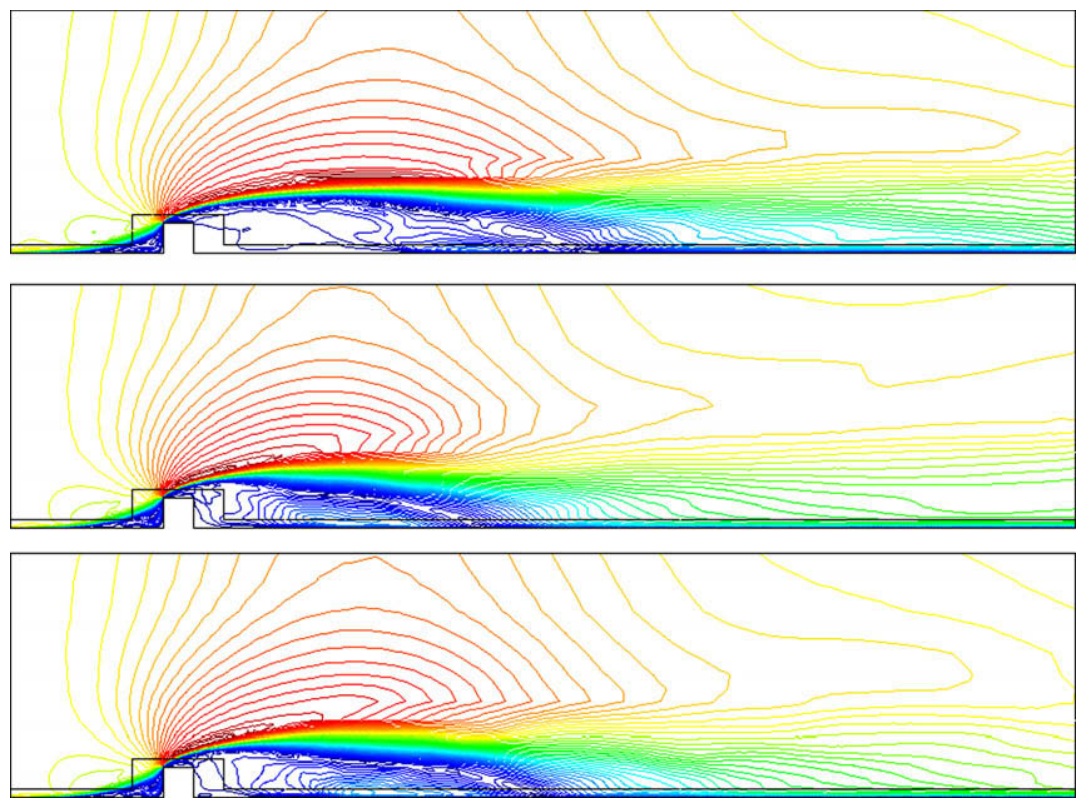

Fig. 4. Averaged velocity field obtained with LES (top), with QSS (middle) and with DS (bottom). 
enters the domain on the left side at $x=-5$ in the positive $x$-direction which is subsequently referred to as the streamwise direction. No-slip boundary conditions are applied on the bottom surface at $y=-1$ and on the remaining three surfaces of the obstacle. The top of the domain at $y=7$ is modeled as impermeable, meaning that the velocity component normal to the surface is set to zero. Periodic boundary conditions are prescribed for the lateral sides at $z=0$ and $z=4$. The outflow at $x=30$ is modeled with the free outflow boundary condition.

The spatial discretization is performed using a structured mesh near the walls (except around the obstacle) in combination with an unstructured mesh elsewhere on each $x-y$ plane. Since the flow is homogeneous in the $z$-direction, the domain width is divided in 32 elements, resulting in a rather coarse mesh of around $2.2 \mathrm{M}$ ele- ments. Near the obstacle the resolution achieved is almost that of a DNS [29] and near the walls it is also very good, being rather coarse in the shear layer region (see below). A detail of the mesh around the obstacle is shown in Fig. 3.

Three different numerical simulations were carried out. The first one was performed using the standard Smagorinsky model and the stabilized finite element formulation of Codina et al. [9] considering the subscales quasi-static, that is to say, dropping their time derivative in the formulation presented above. It is referred to as LES in the following. The second one was performed using just the stabilized formulation of Codina et al. [9] considering quasistatic subscales without additional LES modeling. Below, it is referred to as QSS. The last one was performed using the stabilized finite element formulation of Codina et al. [9] considering time

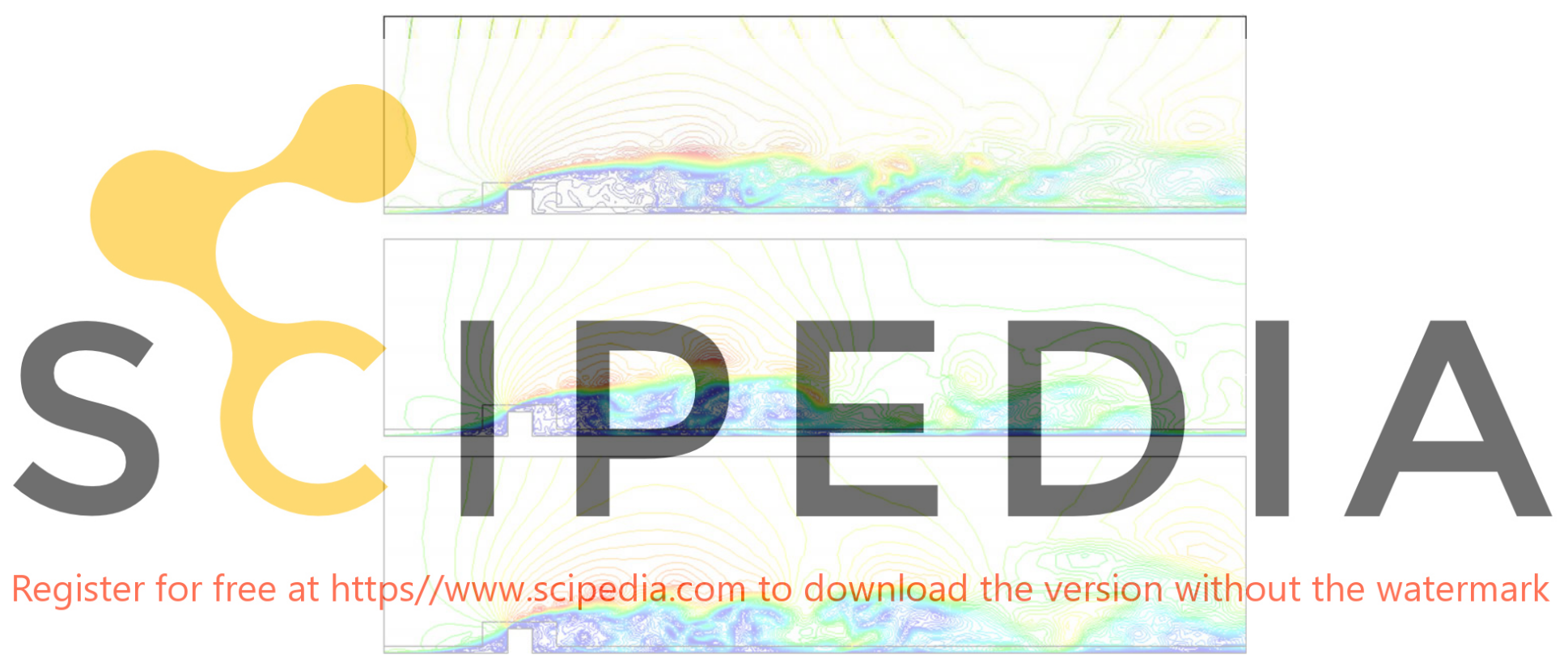

Fig. 5. Instantaneous velocity field obtained with LES (top), with QSS (middle) and with DS (bottom).
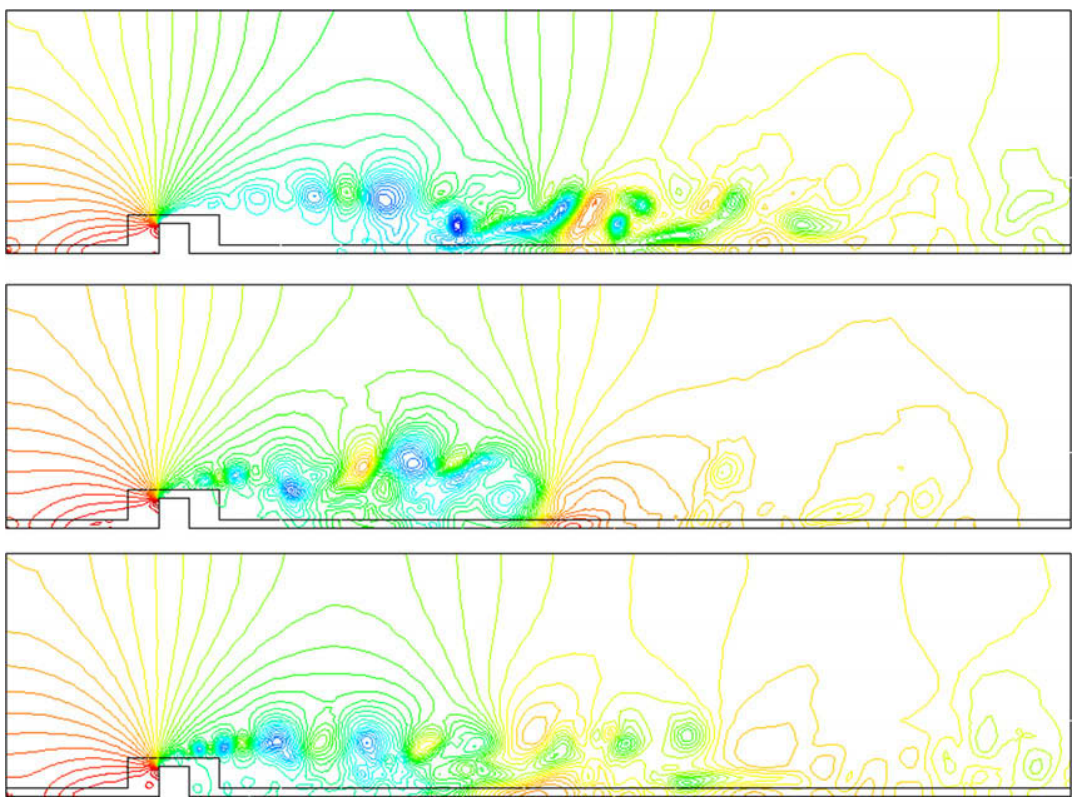

Fig. 6. Instantaneous pressures obtained with LES (top), with QSS (middle) and with DS (bottom). 
Table 1

Reattachment lengths for different methods

\begin{tabular}{llll}
\hline Method & \multicolumn{2}{l}{ Separation bubble } & Reattachment \\
\cline { 2 - 3 } & {$[\delta x / h]$} & {$[\delta y / h]$} & {$\left[X_{\mathrm{R}} / h\right]$} \\
\hline LES & 2.46 & 0.723 & 14.32 \\
QSS & 2.71 & 0.724 & 10.63 \\
DS & 2.70 & 0.725 & 12.75 \\
LES [1] & 2.5 & 0.75 & 15.42 \\
LES [25] & 1.9 & & 12.8 \\
DNS [25] & 2.0 & & 13.2 \\
\hline
\end{tabular}

dependent subscales without additional LES modeling. It is the one labeled DS in the following.

Starting from a lower Reynolds number solution the flow is evolved until a statistically steady state is reached. This occurs at approximately 10 residence times (the time a fluid particle stays inside the domain) and statistical data is accumulated for another 10 residence times. Averaging for velocities is performed both in the $z$-direction and in time, whereas for the dissipations to be discussed later it is only performed in time and plotted at $z=4$. In both cases, though, it is denoted by

The physics of the problem has much in common with that of the backward facing step problem [22], the main difference being the separation zone upstream of the obstacle. Due to this separation bubble, the flow does not separate at the rear edge of the obstacle, where the separation line of the backward-facing step is located, but at the front edge. The separated shear layer is net horizontal, but includes an angle with the top surface of the abstacle enclosing a recirculation region.

The free separated shear layer expands, becornes wavy and eventually reattaches at the bottom wall of the domain. However, this reattachment process is very unsteady. The shear layer flaps and large vortical structures shed from it, which swirl the recirculation zone and the fluid they pass on their way to the outflow. These structures are associated with large velocity fluctuations in the z-direction. This pattern is observed in more or less periodic time intervals. Only time averaging of the flow field reveals the picture of an arc-shaped shear layer with a quiet recirculation zone and a stable reattachment point as it is seen in Fig. 4. Instantaneous velocities are shown in Fig. 5 and instantaneous pressures in Fig. 6.
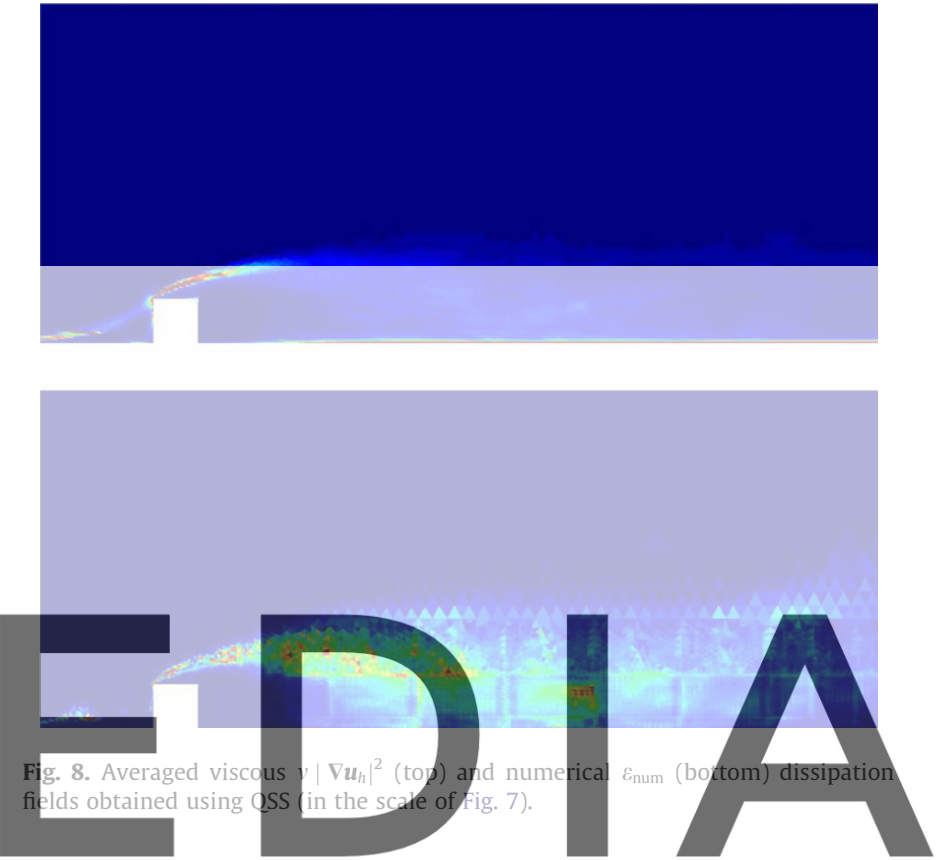

Register for free at https//www.scipedia.com to download the version without the watermark
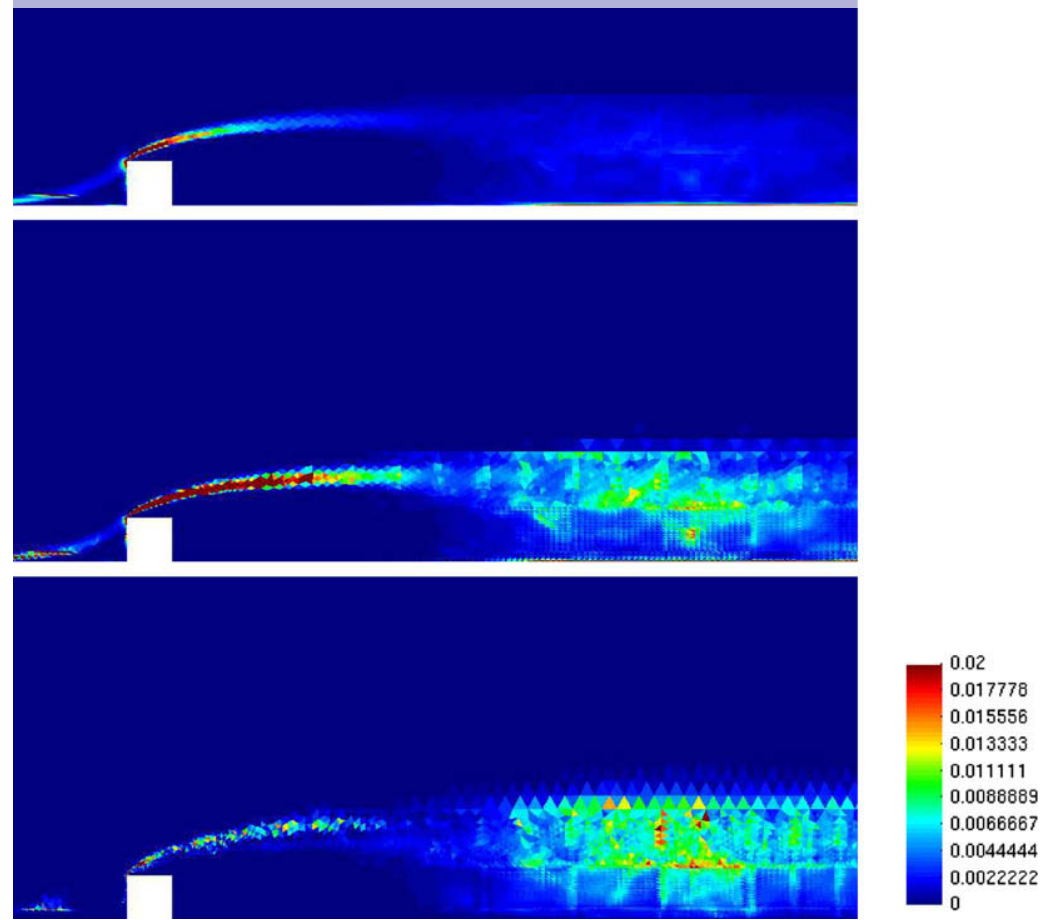

Fig. 7. Averaged viscous $v\left|\nabla \boldsymbol{u}_{h}\right|^{2}$ (top), turbulent $v^{t}\left|\nabla \boldsymbol{u}_{h}\right|^{2}$ (middle) and numerical $\varepsilon_{\text {num }}$ (bottom) dissipation fields obtained using LES. 

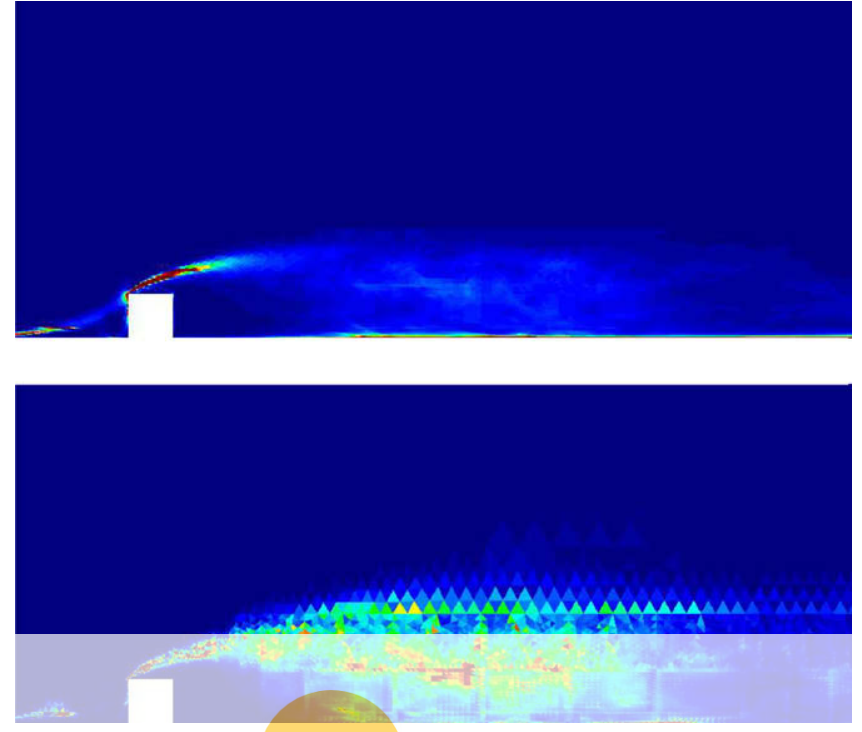

Fig. 9. Averaged viscous $v\left|\nabla \boldsymbol{u}_{h}\right|^{2}$ (top) and numerical $\varepsilon_{\text {num }}$ (bottom) dissipation fields obtained using DS (in the scale of Fig. 7).

The structure of the recirculation zone actually consists of a system of three recirculations. First there is the big one, bounded by the shear layer and the reattachment point. Close to the obstacle
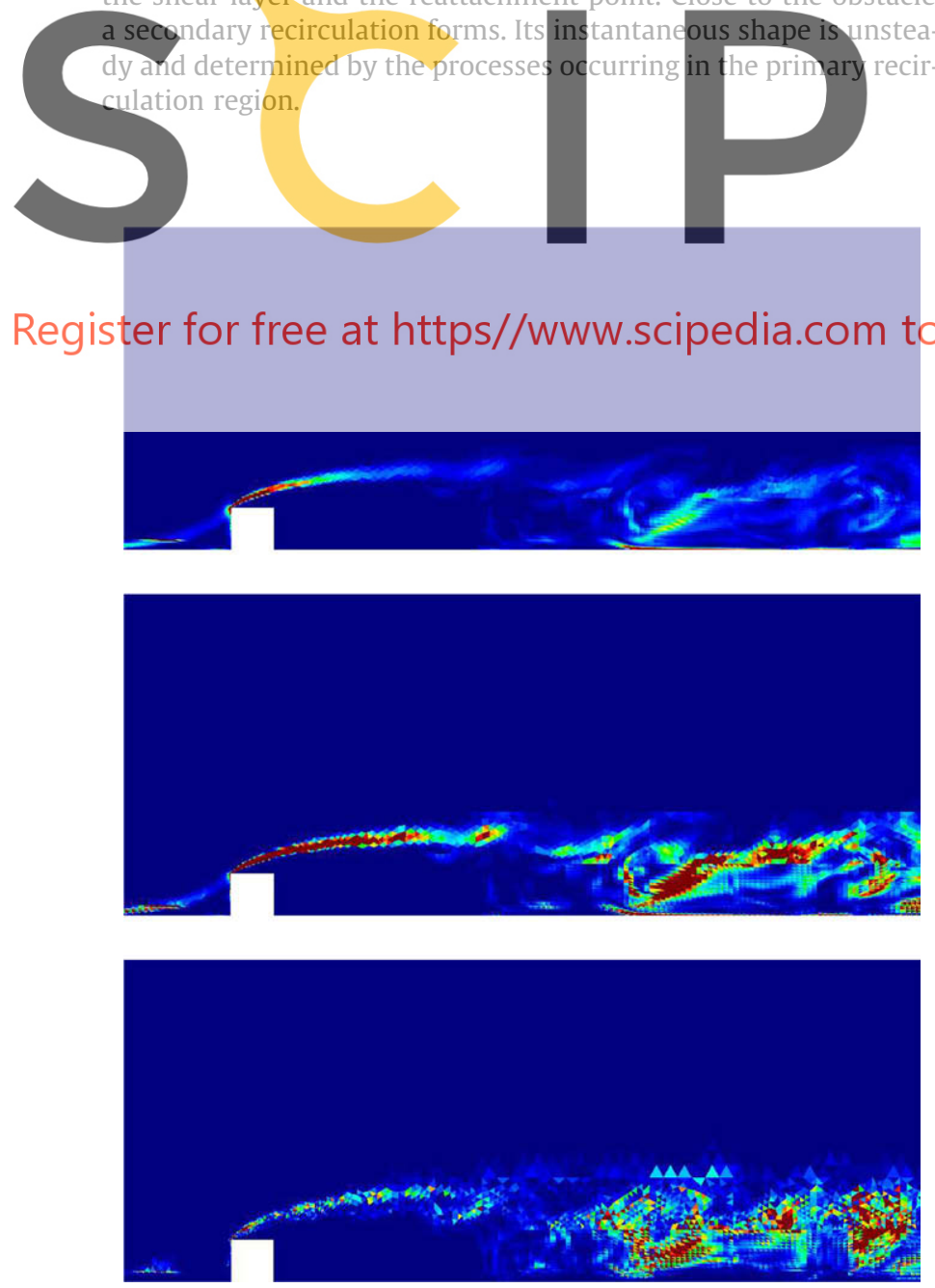

Fig. 10. Instantaneous viscous $v\left|\nabla \boldsymbol{u}_{h}\right|^{2}$ (top), turbulent $\boldsymbol{v}^{t}\left|\nabla \boldsymbol{u}_{h}\right|^{2}$ (middle) and numerical $\varepsilon_{\text {num }}$ (bottom) dissipation fields obtained using LES (in the scale of Fig. 7).
The most important parameter inherent to separated-reattached flows is the reattachment length, denoted by $X_{\mathrm{r}}$. In case of the flow over a surface-mounted obstacle $X_{\mathrm{r}}$ is defined as the length of the recirculation zone downstream of the obstacle. In Table 1 the reattachment length is shown together with the size of the separation bubble upstream the obstacle ( $\delta x$ being the length and $\delta y$ the height). As pointed out in [1], the values obtained are within the range of published experimental results. The reattachment length obtained using LES is in good agreement with that obtained in [1], whereas the one obtained using DS is closer to the LES and DNS of [25]. The reattachment length obtained using QSS is the lowest one.

It is not our intention here to present a complete description of the flow field (see [1]) but rather to highlight the influence the turbulent and numerical dissipation have on it, particularly in the reattachment length. Future work will be devoted to a detailed comparison of the results obtained with the different

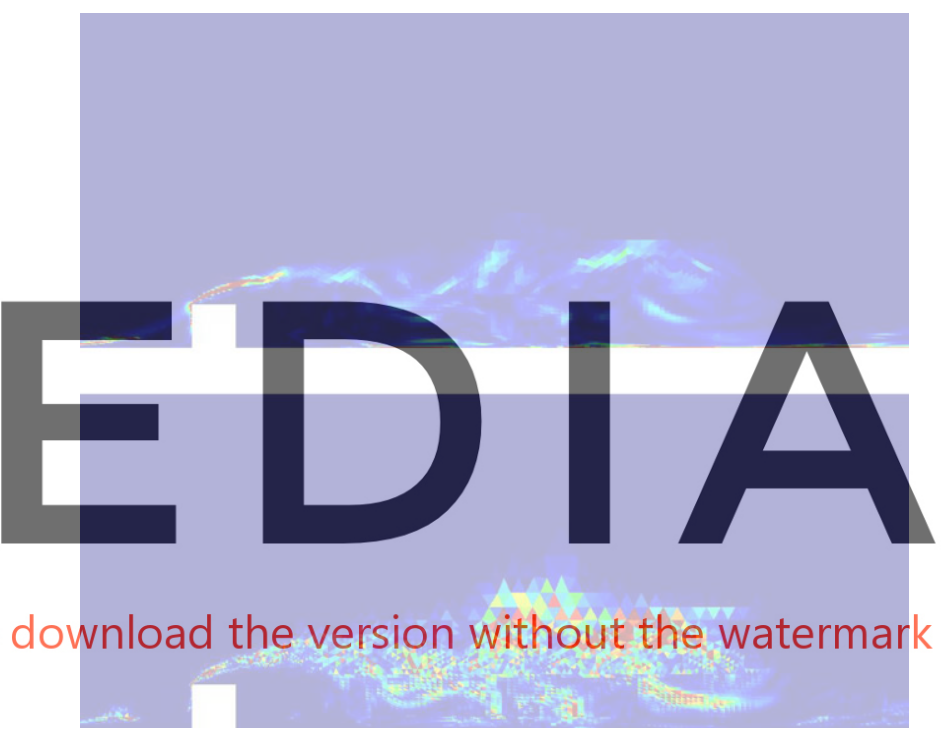

Fig. 11. Instantaneous viscous $\boldsymbol{v}\left|\boldsymbol{\nabla} \boldsymbol{u}_{h}\right|^{2}$ (top) and numerical $\varepsilon_{\text {num }}$ (bottom) dissipation fields obtained using QSS (in the scale of Fig. 7).
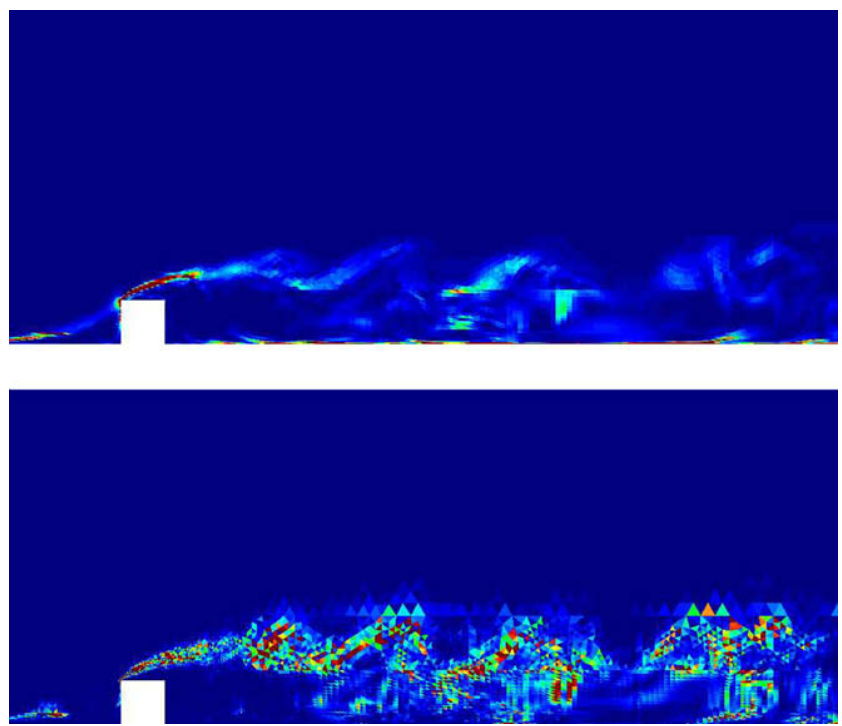

Fig. 12. Instantaneous viscous $v\left|\nabla \boldsymbol{u}_{h}\right|^{2}$ (top) and numerical $\varepsilon_{\text {num }}$ (bottom) dissipation fields obtained using DS (in the scale of Fig. 7). 


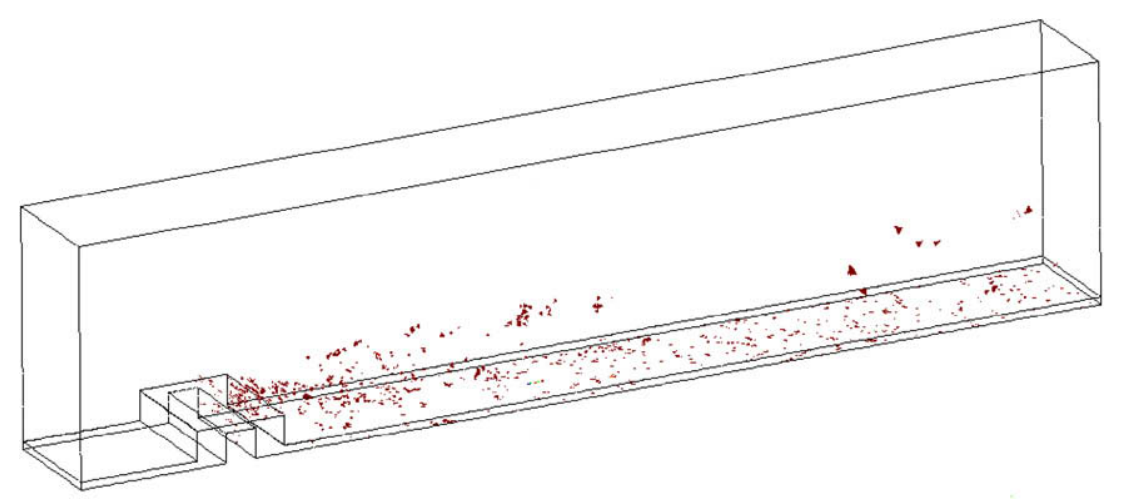

Fig. 13. Points with negative numerical dissipation using DS.

methods used here and the influence of dissipation on turbulence statistics.

Averaged values of viscous, turbulent and numerical dissipation obtained using LES are shown in Fig. 7, whereas averaged viscous and numerical dissipations obtained using QSS and DS are shown in Figs. 8 and 9, respectively. The beginning of the shear layer, near the front edge of the obstacle where the flow separates, is the zone of higher velocity gradients as can be seen in these figures. The viscous dissipation is comparable to the numerical or the turbulent one in the shear layer and in the boundary layer and it is noticeably smaller in the rest of the domain. As discussed

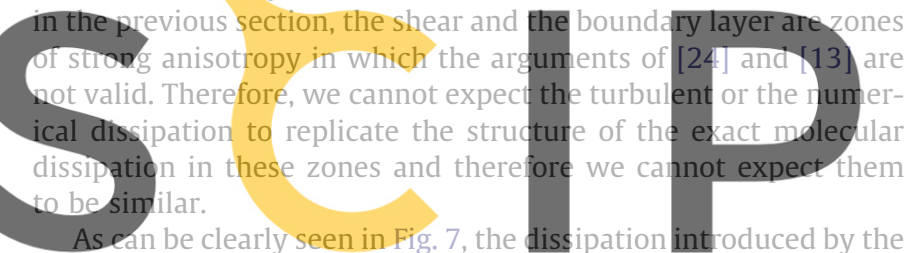
Smagorinsky model is similar to the numerical dissipation, except in the shear and boundary layers. In the shear layer zone the tur-

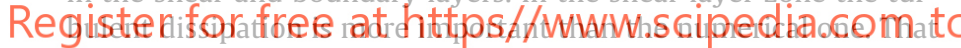
could be explained by the dependence of the eddy viscosity with the velocity gradients, which are very high there. As a consequence, the shear layer is stabilized and unsteady motions generated by the flapping start further downstream. The effect of stabilization of the shear layer can be seen comparing the mean velocities in Fig. 4 and the instantaneous velocities in Fig. 5 obtained with and without LES. This explains why the reattachment length obtained using LES is greater than the ones obtained using QSS and DS. In the boundary layer the turbulent dissipation is higher than the numerical one, which is almost negligible, as seen in Fig. 7. This is a well known misbehavior that is corrected using a dumping function (not used in this work). It is not easy to determine how important is the influence of this dissipation in the reattachment length although it is reasonable to think that it will influence the shear stress at the wall.

In the rest of the domain, both the turbulent and the numerical dissipation have a similar spatial structure, the latter showing a slightly more pronounced dependence on the mesh size in the zone where bigger elements are observed. We recall that a similar spatial structure should be observed only when $h^{-1}$ lies in the inertial range. In any case, numerical values are similar and this can be seen as an indication of the statistical equivalence between the turbulent and the numerical dissipations to the physical dissipation. As it has been mentioned in the previous section we should have

$\left\langle v|\nabla \boldsymbol{u}|^{2}\right\rangle \quad$ (exact physical dissipation)

$$
\begin{aligned}
& \sim\left\langle v^{t}\left|\nabla \boldsymbol{u}_{h}\right|^{2}\right\rangle \quad \text { (LES dissipation) } \\
& \sim\left\langle\varepsilon_{\text {num }}\right\rangle \quad \text { (numerical dissipation) }
\end{aligned}
$$

where the symbol $\sim$ has to be understood as an approximation up to constants. Of course, this is only an indication as we do not know the exact solution $\boldsymbol{u}$, which can only be obtained by DNS.

When the Smagorinsky model is not used the total dissipation in the shear layer is smaller and therefore unsteady flapping and vortex generation is observed closer to the obstacle. The spatial structure of the numerical dissipation is now more spread than in the LES simulation, as it can be observed in Fig. 8 and in Fig. 9, producing smaller reattachment lengths, as discussed above. It is difficult to analyze why the reattachment length obtained when the quasistatic subscales are used is smaller than the one obtained

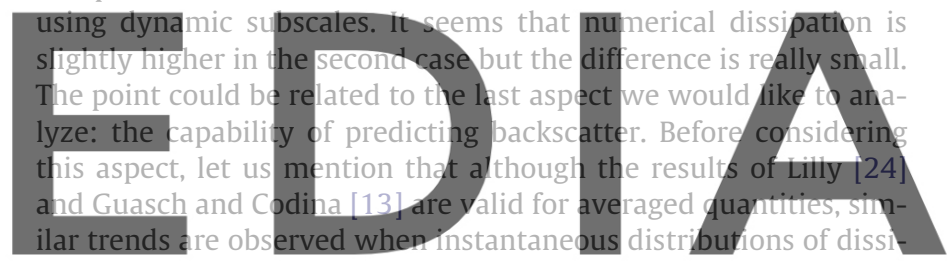

pation are observed. Figs. 10-12 display the viscous and numerical dissipations for the three approaches analyzed at a certain time in-

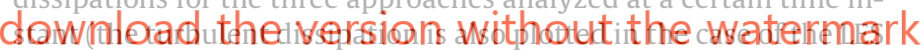
model)

Finally, we have mentioned the possibility to predict backscatter. We have numerically checked that there are element domains in which the dissipation given by (17) is negative, demonstrating that our method has the potential for exhibiting backscatter. When averaging in time or space, the result becomes always positive, but at some points and at some time instants the values of $\varepsilon_{h}$ may be negative. A snapshot of this situation is shown in Fig. 13. As it can be observed there, these points are mainly located close to the boundary layer and the shear layer. Again, a DNS would be the only way to check that the spatial distribution observed in Fig. 13 actually represents the physical situation.

\section{Conclusions}

In this paper we have discussed the dissipative structure of a two-scale finite element approximation for incompressible flow problems. A discussion of what is dissipation in our formulation, which terms account for it and their physical meaning has been the main contribution of this paper.

As particular features of our approach, we have considered the subgrid scales time dependent (dynamic) and orthogonal to the finite element space. These features have proved to be essential to draw the main conclusions of the paper:

- Only when the space of subscales is orthogonal to the finite element space there is a proper scale separation between finite element scales and subgrid scales in the energy balance equation. 
We have used the term "proper scale separation" meaning that the variation of the total kinetic energy is the sum of the variations of the kinetic energy of the two scales, and also the total dissipation is the sum of the dissipations of the two scales.

- The structure of the numerical dissipation, and in particular of the transfer of energy from the finite element scales to the subscales, has been examined in a numerical experiment. This numerical dissipation, and also the turbulent one, are known to be statistically proportional to the (exact) molecular dissipation when the flow is locally isotropic. It is observed in the numerical experiment that these dissipations have a similar spatial structure except in the zones of strong anisotropy. In the boundary and shear layers the dissipation coming from a LES model (the simplest Smagorinsky model, in our case) is higher than the numerical one.

- The possibility of having backscatter has been effectively checked in the numerical experiments presented. This backscatter appears temporarily where it is known it could appear, namely, close to boundary and shear layers and in rather isolated spots. Noteworthy, backscatter is a feature possible only when dynamic subgrid scales are considered.

All these conclusions point to the direction we currently favor, and it is that the two scale numerical approach presented herein, splitting the unknown into the finite element component and a subscale, is able to model turbulence. This does not imply at all that our formulation cannot be improved, and in particular "closures" for the subgrid scale other than the one followed here can be certainly devised. Nevertheless, the clean dissipative structure of the formulation described is one additional argument in favor of the position to consider turbulence modeling a numerical issue.

\section{References}

[1] I.E. Abdalla, M.J. Cook, Z. Yang, Numerical study of transitional separatedreattached flow over surface-mounted obstacles using large-eddy simulation, Int. J. Numer. Methods Fluids 54 (2) (2007) 175-206.

[2] Y. Bazilevs, V.M. Calo, J.A. Cottrell, T.J.R. Hughes, A. Reali, G. Scovazzi, Variational multiscale residual-based turbulence modeling for large eddy simulation of incompressible flows, Comput. Method. Appl. Mech. Engrg. 197 (1-4) (2007) 173-201.

[3] J.P. Boris, F.F. Grinstein, E.S. Oran, R.L. Kolbe, New insights into large eddy simulation, Fluid Dyn. Res. 10 (1992) 199.

[4] E. Burman, Interior penalty variational multiscale method for the incompressible Navier-Stokes equation: monitoring artificial dissipation, Comput. Method. Appl. Mech. Engrg. 196 (41-44) (2007) 4045-4058.

[5] V.M. Calo, Residual based multiscale turbulence modeling: finite volume simulations of bypass transition, PhD Thesis, Department of Civil and Environmental Engineering, Stanford University, 2004.

[6] R. Codina, A stabilized finite element method for generalized stationary incompressible flows, Comput. Method. Appl. Mech. Engrg. 190 (20-21) (2001) 2681-2706.
[7] R. Codina, Stabilized finite element approximation of transient incompressible flows using orthogonal subscales, Comput. Method. Appl. Mech. Engrg. 191 (39-40) (2002) 4295-4321.

[8] R. Codina, J.M. González-Ondina, G. Díaz Hernández, J. Principe, Finite element approximation of the modified Boussinesq equations using a stabilized formulation, Int. J. Numer. Meth. Fl. 57 (9) (2008) 1249-1268.

[9] R. Codina, J. Principe, O. Guasch, S. Badia, Time dependent subscales in the stabilized finite element approximation of incompressible flow problems, Comput. Method. Appl. Mech. Engrg. 196 (21-24) (2007) 2413-2430.

[10] J.A. Domaradzki, Z. Xiao, P.K. Smolarkiewicz, Effective eddy viscosities in implicit large eddy simulations of turbulent flows, Phys. Fluids 15 (12) (2003) 3890-3893.

[11] E. Garnier, M. Mossi, P. Sagaut, P. Comte, M. Deville, On the use of shockcapturing schemes for large-eddy simulation, J. Comput. Phys. 153 (2) (1999) 273-311.

[12] S. Ghosal, An analysis of numerical errors in large-eddy simulations of turbulence, J. Comput. Phys. 125 (1) (1996) 187-206.

[13] O. Guasch, R. Codina, A heuristic argument for the sole use of numerical stabilization with no physical LES modelling in the simulation of incompressible turbulent flows, J. Comput. Phys., submitted for publication.

[14] J. Hoffman, C. Johnson, A new approach to computational turbulence modeling, Comput. Method. Appl. Mech. Engrg. 195 (2006) 2865-2880.

[15] T.J.R. Hughes, G. Engel, L. Mazzei, M.G. Larson, The continuous Galerkin method is locally conservative, J. Comput. Phys. 163 (2) (2000) 467-488.

[16] T.J.R. Hughes, L.P. Franca, G. Scovazzi, Multiscale and stabilized methods, Encyclopedia Comput. Mech. 3 (2004).

[17] T.J.R. Hughes, L. Mazzei, A.A. Oberai, The multiscale formulation of large eddy simulation: decay of homogeneous isotropic turbulence, Phys. Fluids 13 (2) (2001) 505-512.

[18] T.J.R. Hughes, A.A. Oberai, L. Mazzei, Large eddy simulation of turbulent channel flows by the variational multiscale method, Phys. Fluids 13 (6) (2001) 1784-1799.

[19] T.J.R. Hughes, V.M. Calo, G. Scovazzi, Variational and multiscale methods in turbulence, in: W. Gutkowski, T.A. Kowalewski (Eds.), Proceedings of the XXI International Congress of Theoretical and Applied Mechanics (IUTAM), Kluwer, 2004.

[20] T.J.R. Hughes, G.R. Feijóo, L. Mazzei, J.B. Quincy, The variational multiscale method - a paradigm for computational mechanics, Comput. Method. Appl. Mech. Engrg. 166 (1998) 3-24.

[21] A.G. Kravchenko, P. Moin, On the effect of numerical errors in large eddy simulations of turbulent flows, J. Comput. Phys. 131 (2) (1997) 310-322.

[22] H. Le, P. Moin, J. Kim, Direct numerical simulation of turbulent flow over a backward-facing step, J. Fluid Mech. 374 (1997) 330-349.

[23] V. Levasseur, P. Sagaut, F. Chalot, A. Davroux, An entropy-variable-based VMS/ GLS method for the simulation of compressible flows on unstructured grids, Comput. Method. Appl. Mech. Engrg. 195 (9-12) (2006) 1154-1179.

[24] D.K. Lilly, The representation of small-scale turbulence theory in numerical simulation experiments, in: H.H. Goldstine (Ed.), Proceedings of the IBM Scientific Computing Symposium on Environmental Sciences, 1967.

[25] A. Orellano, H. Wengle, Numerical simulation (dns and les) of manipulated turbulent boundary layer flow over a surface-mounted fence, Eur. J. Mech. BFluid. 19 (5) (2000) 765-788.

[26] P. Sagaut, Large Eddy Simulation for Incompressible Flows, Scientific Computing, Springer, 2001.

[27] P.A.B. Sampaio, P.H. Hallak, A.L.G.A. Coutinho, M.S. Pfeil, A stabilized finite element procedure for turbulent fluid-structure interaction using adaptive time-space refinement, Int. J. Numer. Meth. Fl. 44 (2004) 673-693.

[28] A.E. Tejada-Martínez, K.E. Jansen, On the interaction between dynamic model dissipation and numerical dissipation due to streamline upwind/PetrovGalerkin stabilization, Comput. Method. Appl. Mech. Engrg. 194 (9-11) (2005) 1225-1248.

[29] A. Yakhot, H. Liu, N. Nikitin, Turbulent flow around a wall-mounted cube: a direct numerical simulation, Int. J. Heat Fluid Fl. 27 (6) (2006) 994-1009. 